\title{
Sportive Education at Rome. \\ Campus and Religious Practices L'ÉDUCATION SPORTIVE À ROME. CAMPUS ET PRATIQUES RELIGIEUSES
}

Catherine WolfF

Université d’Avignon et des Pays de Vaucluse

CATHERINE.WOLFF@UNIV-AVIGNON.FR

\section{RÉSUMÉ}

Pour les jeunes gens de l'élite, à Rome, la formation intellectuelle devait être complétée par une formation physique. Cette éducation sportive (maniement des armes, équitation et natation pour l'essentiel) prenait place sur le Champ de Mars à Rome et sur le campus dans les villes d' Italie et des provinces occidentales. Le campus était aussi le terrain d'exercice des soldats, et ces derniers honoraient des divinités particulières qui protégeaient le campus. Elles n' apparaissaient pas sur les campus civils.

\section{Abstract}

A physical training was supposed to complete the intellectual training of the elite youth in Rome. This sports education (mostly arms drill, riding and swimming) took place on the Campus Martius in Rome and in the campus for the Italian cities and the cities of the occidental provinces. The campus was also the training field of the soldiers. They worshipped particular deities, who protected the military campus but were not present on the civil campus. 
MOTS CLÉS

Éducation sportive, jeunesse, campus, campestres.

\section{KEY WORDS}

Sportive education, youth, campus, campestres. 
L' EXERCICE PHYSIQUE ÉTAIT TRÈS IMPORTANT À ROME. Il était le complément attendu de la formation intellectuelle et le jeune Romain ne recevait pas une éducation complète s'il ne le pratiquait pas. Même les auteurs qui, comme Quintilien ou Sénèque, voyaient l'exercice physique comme un concurrent possible de l'activité intellectuelle et recommandaient de privilégier la seconde plutôt que le premier n'en soulignaient pas moins la nécessité de ce dernier, à condition donc qu’il fût modéré ${ }^{1}$ Quintilien ne peut que préconiser à l'orateur d'entretenir son corps. Prononcer un discours est en effet aussi un exercice physique et l'orateur doit par conséquent veiller à la bonne santé de son corps. Mais l'entraînement physique ne saurait être un but en soi².

Cet exercice physique n'était pas conçu comme celui des Grecs. Cicéron évoque par l'intermédiaire de Scipion l'absurdité de l'entraînement des jeunes au gymnase et le caractère inapproprié de l'entraînement militaire des éphèbes ${ }^{3}$. On trouve déjà ce second reproche chez des auteurs grecs, mais cela n'en éclaire pas moins la vision romaine des exercices pratiqués par les Grecs ${ }^{4}$. Un autre reproche très fréquent

1. Quint., Inst., I, 11, 15-17 ; Sen., Ep., II, 15, 2-4 et 6 ; X, 83, 3-4 ; XI-XIII, 88, 19.

2. GRODDE, O. : Sport bei Quintilian, Hildesheim, Weidmann, 1997, 72-87.

3. Cic., Rep., IV, 4. Même idée chez Luc., VII, 270-272. BOOTH, A.D. : « Roman Attitudes to Physical Education », EMC, 19, 1975, 27-34 (27) ; CROWTHER, N.B. : « The Palaestra, Gymnasium and Physical Exercise in Cicero », Nikephoros, 15, 2002, 159-174 (174). WALLNER, C. : « Cuncta pudenda confluunt celebranturque. Griechische Athleten in Rom », P. Mauritsch (éd.), Körper im Kopf. Antike Diskurse zum Körper, Graz, Grazer Universitätsverlag, 2010, 131-156 (138-148) et MAMMEL, K. : « Ancient Critics on Roman Spectacle and Sport ", P. Christesen et D.G. Kyle (éd.), A companion to sport and spectacle in Greek and Roman Antiquity, Malden/Oxford, Blackwell, 2013, 603-615 pour une revue de l'ensemble des critiques des auteurs romains à l'encontre de l'entraînement physique «à la grecque ". Pour K. Mammel, ces critiques émanent des membres de l'élite, des médecins et des intellectuels et sont le reflet de leurs préoccupations et de leurs a priori.

4. CROWTHER, N.B. : "Cicero's attitude to Greek athletics ", Nikephoros, 14, 2001, 63-81 (67). Voir aussi MANN, C. : "Griechischer Sport und römische Identität : die certamina athletarum in Rom ", 
à l'encontre de l'exercice physique tel qu'il était pratiqué par les Grecs était celui de l'immoralité et de la corruption qu' il entraînait pour ceux qui s'y adonnaient ${ }^{5}$. Comme l'écrit Plutarque, les Romains étaient très méfiants à l'égard de l'usage de l' huile à sec et ils considéraient que c'étaient les gymnases et les palestres qui avaient causé l' amollissement et l'asservissement des Grecs ${ }^{6}$. Le discours des adversaires de Scipion l'Africain est très caractéristique à cet égard. Quand ils décrivent son comportement à Syracuse, ils déclarent qu'il ne se conduit ni comme un Romain ni comme un soldat : il est vêtu à la grecque, il fréquente le gymnase et s'applique aux exercices de la palestre ${ }^{7}$. Les Grecs, selon Plutarque, de " vaillants hommes d' armes » et " brillants cavaliers » qu' ils avaient été, étaient devenus des " athlètes bien faits " et des " champions de palestre ${ }^{8}$. Il y avait donc d'un côté pour les Romains, tout ce qui entraînait au maniement des armes et à l'équitation, et de l'autre tout ce qui était pratique sportive athlétique, qui ne permettait pas de devenir un bon soldat ${ }^{9}$. Aux yeux des Romains, la nudité liée aux exercices gymniques était une des causes de cette immoralité et de cette corruption et elle les gênait tout particulièrement ${ }^{10}$. Le deuxième reproche proprement romain à l'encontre des pratiques grecques concernait le statut social de ceux qui prenaient part aux compétitions : des membres de l'élite dans les cités grecques. Pour les Romains, c'étaient les esclaves, les affranchis ou au mieux les citoyens romains des couches inférieures de la population qui descendaient dans l'arène ou le cirque ${ }^{11}$. Enfin les médecins comme Galien soulignaient les effets néfastes de l'exercice physique intensif et du régime suivi par les athlètes

Nikephoros, 15, 2002, 147-148 ; GARCÍA ROMERO, F. : « Ancient Greek sport and Roman identity », B. Kratzmüller, M. Marschik, R. Müllner, H.D. Szemethy, E. Trinkl (éd.), Sport and the construction of identities, Vienne, Turia + Kant, 2007, (443-452) 445-448.

5. GARCÍA ROMERO, F. : art. cit., 443-444 et 446-447.

6. Plu., Quaest. Rom., 40, 274d.

7. Liv., XXIX, 19, 11-12. Plutarque, Cato Ma., 3, 7, met dans la bouche de Caton l'Ancien les mêmes accusations à l'encontre de Scipion l'Africain.

8. Plu., Quaest. Rom., 40, 274d. Str., X, 4, 16, à propos des mesures prises par Éphore en Crète ; Tac., Ann., XIV, 20. GARCÍA ROMERO, F. : art. cit., 444.

9. MANN, C. : art. cit., 125-158 : le discours négatif des élites concernant l'athlétisme grec est utilisé pour mettre en avant l' identité romaine et l'opposer à l'altérité grecque.

10. Cic., Ver., II, 5, 72, 185 ; Cael., XV, 36 ; Arch., VI, 13 ; Tusc., IV, 33, 70 ; Hor., S., II, 2, 11 ; Ars, 380 ; Carm., III, 7, 26 ; Prop., III, 14, 6 ; 10 ; Sen, Ep., XI-XIII, 88, 3-5 ; Plin., Ep., II, 17. Daremberg-Saglio, s.v. Educatio, 487 (E. Pottier) ; CROWTHER, N.B. : " Nudity and morality : Athletics in Italy », Classical Journal, 76, 1980-1981, 119-123 = Athletika. Studies on the Olympic Games and Greek athletics, Hildesheim, Weidmann, 2004, 375-379; REIS, M. : Sport bei Horaz, Hildesheim, Weidmann, 1994, 12 ; THUILLIER, J.-P. : « Le corps du sportif romain », P. Moreau (éd.), Corps romains, Grenoble, Jérôme Millon, 2002, 251-266; CORDIER, P. : « Gymnase et nudité à Rome ", Et si les Romains avaient inventé la Grèce, Mètis, n.s. 3, 2005, 253-269 (256-265) ; GARCÍA ROMERO, F. : art. cit., 447.

11. MANN, C. : art. cit., 125, 131-135 et 147-151.

ARYS, 15, 2017 [171-186] ISSN 1575-166X 
sur le corps et souhaitaient que les médecins fussent plus écoutés que les entraîneurs par les athlètes ${ }^{12}$. La pratique de la gymnastique a toutefois progressivement pris de l' importance, dans les palestres des thermes en particulier, et les Romains sont devenus familiers des pratiques sportives grecques ${ }^{13}$. Quand Quintilien aborde la question de la pratique sportive pour le futur orateur, c'est à un palaestricus qu' il pense pour donner des leçons à ce dernier ${ }^{14}$. Il n'y a cependant jamais eu beaucoup d'athlètes professionnels romains et les concours "à la grecque ", avec boxe, lutte, pancrace ${ }^{15}$, pentathlon et courses, sont restés relativement peu nombreux à Rome. Suivant en cela l'exemple d'Auguste, certains empereurs comme Néron avec les Neronia (célébrés en 60 et 65 uniquement) ou Domitien avec les Capitolia, les jeux capitolins, ont cherché à mettre à l' honneur en Occident ce type d'épreuves ${ }^{16}$. Certes les Capitolia, institués en 86 en l' honneur de Jupiter Très Bon Très Grand, ont continué à être organisés jusqu'au $\mathrm{IV}^{\mathrm{e}}$ siècle apr. J.-C., mais ce type de tentatives n'a guère fait d'émules à Rome, alors que les empereurs ont encouragé l'organisation et la pratique de ces concours dans l'Orient grec $^{17}$.

12. FELSENHELD, É. : « Galien et la gymnastique : science sans conscience n'est que ruine de l'âme », BAGB, 2, 2009, 131-145 ; MAMMEL, K.: art. cit., 608.

13. CROWTHER, N.B. : art. cit., 1980-1981 [2004], 379 ; REIS, M. : ouv. cit., 12 ; FORTUIN, R. : Der Sport im augusteischen Rom, Stuttgart, Peter Lang, 1996, 22-31 ; LEE, H.M. : " Greek Sports in Rome », P. Christesen et D.G. Kyle (éd.), A companion to sport and spectacle in Greek and Roman Antiquity, Malden/Oxford, Blackwell, 2013, 533-542 (539-540).

14. GRODDE, O. : ouv. cit., 74 et 89.

15. JUNKELMANN, M. : « Griechische Athleten in Rom. Boxen, Ringen und Pankration », E. Köhne et C. Ewigleben (éd.), Caesaren und Gladiatoren. Die Macht der Unterhaltung in antiken Rom, Mayence, P. von Zabern, 2000, 81-90 pour ces trois disciplines, la boxe et la lutte étant pratiquées depuis très longtemps par les Romains.

16. HERZ, P. : « Die Entwicklung der griechischen Agonistik in der Kaiserzeit », N. Müller et M. Messing (éd.), Olympische Studien, Niedernhausen/Taunus, Schors-Verlag, 1988, 111-131 ; GRODDE, O. : ouv. cit., 89; VAN NIJF, O. : « Athletics, festivals and Greek identity in the Roman East », Proceeding of the Cambridge Philological Society, 45, 2000, 176-200 (186-187) ; MANN, C. : art. cit., 140-141 ; WALLNER, C. : art. cit., 132-134; HEINEMANN, A. : « Sportsfreunde : Nero und Domitian als Begründer griechischer Agone in Rom », S. Bönisch-Meyer, L. Cordes, V. Schulz, A. Wolsfeld, M. Ziegert (éd.), Nero und Domitian. Mediale Diskurse der Herrscherrepräsentation im Vergleich, Tübingen, Günter Narr, 2014, 217-263. Pour les précédents républicains et les certamina athletarum à l'époque augustéenne, MANN, C. : art. cit., 136-140 ; CROWTHER, N.B. : « Greek Games in Republican Rome », Antiquité classique, 52, 1983, 268-273 = Athletika. Studies on the Olympic Games and Greek athletics, Hildesheim, Weidmann, 2004, 381-385 ; FORTUIN, R. : ouv. cit., 44, 50-60 et 78-95 ; WALLNER, C. : art. cit., 134-138 ; LEE, H.M. : art. cit., 534-537 et 540 ; HEINEMANN, A. : art. cit., 221-224.

17. VAN NIJF, O. : art. cit., 186-188 ; MANN, C. : art.cit., 141-152, considère que cela ne veut pas dire que ces spectacles n'étaient pas appréciés par le peuple ; PLEKET, H.W. : « Roman Emperors and Greek Athletes ", Nikephoros, 23, 2010, 175-203 (187-199) ; HEINEMANN, A. : art. cit., 218-220 ; GARCÍA ROMERO, F. : art. cit., 448-449. 
En quoi consistait donc l'éducation sportive, le sport (exercitatio) à Rome ? Caton a appris à son fils à lancer le javelot, à combattre en armes et à faire de l'équitation, à pratiquer le pugilat, à supporter le chaud et le froid, à traverser à la nage les tourbillons du Tibre ${ }^{18}$. Cet entraînement très dur pourrait être une spécificité des Porcii, puisque Caton d' Utique endurcit également son corps par un entraînement physique sévère : il s'était habitué à supporter le froid et à marcher en toute saison ${ }^{19}$. Ils n'ont cependant pas été les seuls à agir ainsi. Plaute s' inspire certes des comédies grecques, mais l'énumération qu' il place dans la bouche de l'un de ses personnages, Philolachès, lorsque ce dernier se rappelle avec nostalgie qu' il était le premier à la gymnastique, évoque par de nombreux aspects l'éducation reçue par le fils de Caton : disque, lance, javelot, course, maniement des armes, équitation ${ }^{20}$. Cicéron, lorsqu' il décrit la formation qui conduit à la vertu, mentionne, en même temps que la formation de l'esprit, celle du corps : équitation, chasse, maniement des armes ${ }^{21}$. Chez Horace également, les jeunes gens, quand ils s'entraînent, se livrent à des compétitions et rivalisent entre eux, aussi bien pour l'équitation sur le Champ de Mars que pour la natation dans le Tibre ${ }^{22}$. Léquitation, la chasse, la lutte et la course restent ce qu' il faut louer chez un jeune homme et ce qui est objet de compétition ${ }^{23}$. La pratique de la chasse comme élément de l'éducation sportive est apparue avec Paul-Émile ${ }^{24}$. Grâce à elle, les jeunes gens apprenaient l'équitation et le maniement des armes, c'était donc une façon de se former au métier des armes. La chasse était considérée comme un exercice physique formateur au même titre que l'escrime ou l'équitation. Elle est devenue une activité présentée comme traditionnelle, pratiquée et aimée par les Romains de l'élite ${ }^{25}$. Quand on regrettait les temps présents, qui marquaient la décadence par rapport au passé, on montrait l'enfant qui ne savait plus monter à

18. Plu., Cat. Ma., 20, 6.

19. Plu., Cat. Mi., 5, 6.

20. Pl., Mos., 151-152. BLOOMER, M.W. : The School of Rome. Latin Studies and the Origins of Liberal Education, Berkeley/Los Angeles/Londres, University of California Press, 2011, 33.

21. Cic., Part., XXIII, 80. Voir aussi Cato, 58 : armes, équitation, lancer du javelot, escrime, jeu de balle, natation et course. CROWTHER, N.B.: art. cit., 2002, 174 : Cicéron ne recommande pas le lancer du disque, activité sportive grecque.

22. Hor., Carm., I, 8, 3-12 ; III, 7, 25-28. REIS, M. : ouv. cit., 112-113 : Horace se fait le porte-parole d'Auguste, qui attache beaucoup d' importance à l'entraînement sportif, en particulier à celui des iuuenes.

23. Hor., Carm., III, 12, 7-12.

24. Plb, XXXII, 15, 5-8 (XXXI, 29, 5-8) ; Plu., Aem., VI, 9-10.

25. Hor., Ars, 160-162 ; Ep., I, 18, 49-50 ; HA, Ver., 2, 10. CORBEILL, A. : « Education in the Roman Republic: Creating Traditions ", Y.L. Too (éd.), Education in Greek and Roman Antiquity, Leyde/Boston/ Cologne, Brill, 2001, 261-287 (280-281). 
cheval et n'aimait pas chasser. Il préférait le cerceau grec ou les dés ${ }^{26}$. Hadrien aimait passionnément la chasse ${ }^{27}$ et les empereurs de façon générale sont présentés comme pratiquant des activités physiques traditionnelles : pugilat, lutte, course, chasse, c'est ce qu' aimait Marc Aurèle avant de se consacrer uniquement à la philosophie ${ }^{28}$. Aux mauvais empereurs comme Commode en revanche sont réservés les courses de char et les combats de gladiateurs ${ }^{29}$.

Cette formation physique était donc surtout vue comme une formation aux métiers des armes : au moment où l'on devait attribuer le commandement de la guerre contre Mithridate, Marius, qui souhaitait ce commandement, est allé s'entraîner avec les jeunes gens sur le Champ de Mars, avec maniement des armes et équitation, malgré son âge et son obésité ${ }^{30}$. Végèce énumère les différents exercices physiques pratiqués par Pompée alors qu' il commandait une armée : saut, course, transport. Ses soldats pratiquaient les mêmes exercices, qui les préparaient à la bataille ${ }^{31}$. Il ne s'agit plus de jeunes hommes encore en âge d'aller à l'école, mais de soldats. Une grande partie des exercices était la même : cet entraînement physique était bien une préparation au métier militaire, même si le Champ de Mars était fréquenté aussi par des hommes qui souhaitaient simplement se détendre en jouant à la balle ou au cerceau ${ }^{32}$.

Pour réaliser au mieux la préparation des futurs cadres de l'armée au métier militaire, deux entrainements étaient privilégiés : les armes et l'équitation ${ }^{33}$. Mécène conseille à Auguste de veiller à ce que les enfants des sénateurs et des chevaliers aillent à l'école dès leur enfance, que dès leur adolescence ils reçoivent une éducation physique et qu' ils apprennent à manier les chevaux et les armes ${ }^{34}$. Alors qu' il ne dit rien des professeurs pour la formation intellectuelle, il précise que les professeurs pour la formation physique doivent être employés par l'État et payés par lui. Dans son esprit, cette formation était aussi importante que l' autre, sinon plus, et l'État se devait de la

26. Hor., Carm., III, 24, 51-58.

27. HA, Had., 2, 1.

28. HA, Marc., 4, 9-10. WALLNER, C. : Soldatenkaiser und Sport, Francfort, Peter Lang, 1997, 20 et 229.

29. HA, Com., $2,9$.

30. Plu., Mar., 34, 5-6. FORTUIN, R. : ouv. cit., 11-15.

31. Veg., Mil., I, 9 et de façon plus générale I, 10-11 et 13-18. Voir aussi J., BJ, III, 5, 1 (73) ; HA, Max., 10, 4 ; Veg., Mil., III, 2 pour les exercices et les lieux où les pratiquer en général.

32. Str., V, 3, 8 ; Hor., S., I, 6, 122-126 ; Mart., VII, 72, 9-10 ; XIV, 40 et 47. REIS, M. : ouv. cit., 113 ; KASTNER, M.-O. : «L'enfant et les jeux dans les documents d'époque romaine », BAGB, 1, mars 1995, 85-100 (93-94) ; THUILLIER, J.-P. : Le sport dans la Rome antique, Paris, Errance, 1996, 85 et 88-90 ; CROWTHER, N.B. : art. cit., 2002, 170-171. Pour la valeur diététique de ce genre d'activités, FORTUIN, R. : ouv. cit., 22-31.

33. Suet., Aug., 83, 1 ; Tib., 13, 1 ; Plu., Pomp., 41, 4-5 ; Veg., Mil., I, 18.

34. D. C., LII, 26. 
surveiller. Il s'agissait d'une activité constitutive du citoyen romain puisque durant son séjour à Rhodes, Tibère n'a plus pratiqué le maniement des armes et l'équitation (equi et armorum solitas exercitationes), qu' il pratiquait quotidiennement, et a abandonné le costume romain pour le costume grec ${ }^{35}$.

Le lieu par excellence pour pratiquer ces deux activités était le campus. Suétone présente l'équitation et le maniement des armes comme des exercitationes campestres $^{36}$, qui se pratiquent donc sur le campus, comme les autres exercices physiques, nous l'avons vu ${ }^{37}$. Le lieu d'entraînement à Rome était le Champ de Mars $^{38}$. C'était sur ce Champ de Mars qu'à l'origine les soldats recrutés en mars s'entraînaient en attendant la campagne militaire, en juillet ${ }^{39}$. Même avant la fondation de Rome, c'était dans une plaine (campus), selon Ovide, que Romulus, son frère Remus et leurs compagnons s'entraînaient ${ }^{40}$. Il s'agissait d' une zone libre de constructions, qui se réduisit et se déplaça progressivement vers le nord, en raison de l'activité édilitaire sur le Champ de Mars ${ }^{41}$.

On retrouve ce campus dans de nombreuses villes d'Occident, où les campi sont apparus bien avant la période impériale pour certains ${ }^{42}$. Ce type de monument ne laisse que peu de traces, puisque la plupart du temps il s'agissait d' une aire découverte plate $^{43}$. De taille plus ou moins grande, ce campus était placé, comme le Champ

\section{Suet., Tib., 13, 1.}

36. Suet., Aug., 83, 1.

37. Les autres exercices physiques, tout comme le maniement des armes, pouvaient se pratiquer dans d'autres lieux. Je me limite volontairement au campus, qui est le lieu par excellence de cette pratique.

38. Hor., Ars, 160-162 ; Carm., III, 7, 26 ; Str., V, 3, 8 ; Liv., II, 5 ; D. H., Antiquitates Romanae, V, 13. THUILLIER, J.-P. : ouv. cit., 1996, 81-83 ; BORLENGHI, A. : Campus : organizzazione e funzione di uno spazio pubblico in età romana, Rome, Quasar, 2011,23-24 et 83-104.

39. BORLENGHI, A. : ouv. cit., 23-24 ; LE BOHEC, Y. : « 350000 sportifs professionnels sous le Principat », P. Guisard et C. Laizé (dir.), Le corps, Paris, Ellipses, 2015, 170-187 (174 et 179-180).

40. Ov., Fast., II, 365-368.

41. COARELLI, F. : Guide archéologique de Rome, Paris, Hachette, 1994, 187.

42. DEVIJVER, H. et VAN WONTERGHEM, F. : " Il campus nell' impianto urbanistico delle città romane: testimonianze epigrafiche e resti archeologici », Acta Archaeologica Lovaniensia, 20, 1981, 3368 ; DEVIJVER, H. et VAN WONTERGHEM, F. : « Der 'Campus' der römischen Städte in Italia und im Westen », ZPE, 54, 1984, 195-206; MENNELLA, G. et SPADEA NOVIERO, G. : « Il campus di Albingaunum », MEFRA, 106, 1994, 1, 119-137 ; THUILLIER, J.-P. : ouv. cit., 1996, 91 ; BOUET, A. : Les thermes privés et publics en Gaule narbonnaise, Rome, EFR 320, 2003, t. I, 145.

43. BOUET, A. : « Un nouvel exemple de campus en Gaule Narbonnaise : Vaison-la-Romaine (Vaucluse) », RAN, 31, 1998, 103-117 (111) ; BOUET, A. : " Campus et Juventus dans les agglomérations secondaires des provinces occidentales ", REA, 101, 1999, 461-486 (462) ; BOUET, A. : « Les bâtiments thermaux et sportifs en Aquitaine ", Caesarodunum, 35-36, 2001-2002, 57-75 (61) ; BORLENGHI, A. : ouv. cit., 23 et 27 . 
de Mars à Rome ${ }^{44}$, à l'extérieur de la ville ${ }^{45}$. Virgile devait avoir en tête cette localisation quand il décrit les activités sportives des enfants et des jeunes gens latins : ils s'entraînaient devant la ville (ante urbem $)^{46}$. Les voies qui y conduisaient ou qui le traversaient étaient soigneusement entretenues ${ }^{47}$. Quelques exceptions existent, comme le bâtiment appelé la Grande Palestre à Pompéi, qui se trouve à l'intérieur de la ville, près de l'amphithéâtre ${ }^{48}$. Le campus s'est progressivement monumentalisé. C'est ainsi qu'il pouvait être bordé d'un portique, comme à Herculanum ou Vaison-la-Romaine, il était le plus souvent fermé par une enceinte, il comportait un bassin (piscina) pour la pratique de la natation ${ }^{49}$. On y trouvait, mais plus rarement, des aménagements utilitaires tels que des latrines, comme à Pompéi ${ }^{50}$, ou encore des bains, des exèdres (scholae), un cadran solaire (solarium) ou des lieux de promenade (ambulationes) ${ }^{51}$. Des thermes, un amphithéâtre ou encore le siège de la Iuuentus étaient parfois en relation directe avec lui ou à proximité immédiate ${ }^{52}$. Si c'était l'administration municipale qui s'occupait du campus et de ses aménagements, les

44. Vitr., I, 7, 1.

45. DEVIJVER, H. et VAN WONTERGHEM, F. : art. cit., 1981, 47 ; DEVIJVER, H. et VAN WONTERGHEM, F. : « Neue Belege zum 'Campus' der römischen Städte in Italien und im Westen », ZPE, 60, 1985, 147-158 (149-150) ; BOUET, A. : art. cit., 1999, 462 et 470-472 ; BORLENGHI, A. : ouv. cit., 75 et 178.

46. Verg., A., VII, 162-165. Les activités sportives sont les suivantes : équitation, tir à l'arc, lancer de javelots, course, combat.

47. DEVIJVER, H. et VAN WONTERGHEM, F. : " The campus in the urban organization of Africa and Sardinia : two examples, Carthage and Carales », Africa Romana, X, 1992, 1035-1060 (1036).

48. DEVIJVER, H. et VAN WONTERGHEM, F. : art. cit., 1984, 202 ; GROS, P. : L'architecture romaine, I. Les bâtiments publics, Paris, Picard, 1996, 378. Pour les liens entre le campus et l' amphithéâtre, BORLENGHI, A. : ouv. cit., 75 et 178.

49. BOUET, A. : art. cit., 1999, 462 ; BORLENGHI, A. : ouv. cit., 42-52.

50. BOUET, A. : art. cit., 1999, 462.

51. CIL X, 1236: «Caius Catius fils de Marcus, quattuorvir, s'est occupé de faire aplanir le campus avec l'argent public et s'est occupé de faire faire avec son propre argent un mur d'enceinte, des exèdres, un cadran solaire et un chemin ; au Génie de la colonie et des colons, en raison de l' honneur reçu, que les colons l' utilisent à jamais heureusement ». DEVIJVER, H. et VAN WONTERGHEM, F. : art. cit., 1981, 37-38; DEVIJVER, H. et VAN WONTERGHEM, F. : " Ancora sul campus delle città romane ", Acta Archaeologica Lovaniensia, 21, 1982, 93-98 (93-94) ; DEVIJVER, H. et VAN WONTERGHEM, F. : art. cit., 1984, 199-200 et 204 ; BOUET, A. : art. cit., « Un nouvel exemple», 1998, 111 ; BOUET, A. : art. cit., 1999, 474 ; BOUET, A. : ouv. cit., t. I, 2003, 145 et 285.

52. BOUET, A. : "Complexes sportifs et centres monumentaux en Occident romain : les exemples d'Orange et de Vienne », Revue archéologique, n.s. 1, 1998, 33-105 (47 et 52-57) ; BOUET, A. : art. cit., $1999,462,472$ et 474 . 
notables offraient souvent à leurs concitoyens soit un campus, soit une enceinte pour ce dernier, soit d'autres aménagements qui lui étaient liés ${ }^{53}$.

Les gladiateurs pouvaient s'y entraîner, les citoyens y pratiquer du sport (on pouvait y faire des distributions gratuites d' huile ${ }^{54}$ ), des jeux s'y dérouler ${ }^{55}$. Le campus était l'endroit ubei ludunt ${ }^{56}$, où l'on s'entraînait, cet entraînement n'étant plus nécessairement lié à la guerre, bien qu'il y ait pratique du maniement des armes ${ }^{57}$. Cicéron désigne les exercices pratiqués sur le Champ de Mars par l'expression ludus campestris ${ }^{58}$. Le mot ludus est particulièrement approprié, puisqu'il a été utilisé d'abord pour décrire un entraînement militaire imitant la bataille. De cette notion de caractère artificiel vient le sens de jeu et d'exercice guerrier, puis on est passé à toutes les formes d'instruction, dont intellectuelle, puis à l'endroit où cela avait lieu. Il a fallu alors préciser par une épithète quel était le type d'entraînement pratiqué dans le ludus. D'où l'expression ludus campestris. Le campus était enfin, et peut-être surtout, utilisé par les associations de la Jeunesse, les collegia iuuenum, que l'on trouvait essentiellement en Italie, et les iuuentutes, plutôt rurales et provinciales ${ }^{59}$. Ces associations, remises au goût du jour par Auguste, devaient assurer la formation sportive (maniement des armes et équitation) des jeunes gens appartenant le plus souvent à l'élite $^{60}$. Les futurs héritiers de l'Empire, en tant que princes de la jeunesse, avaient la charge entre autres de favoriser la création dans les provinces des monuments destinés à l'éducation de la jeunesse de l'élite locale, c'est-à-dire des campi. C'est ainsi que

53. DEVIJVER, H. et VAN WONTERGHEM, F. : art. cit., 1981, 45 ; BOUET, A. : art. cit., 1999, 463464 ; BOUET, A. : ouv. cit., 2003, t. II, 178 ; BORLENGHI, A. : ouv. cit., 165-170.

54. CIL V, 5279 : « Lucius Caecilius Cilo, fils de Lucius [...] a ordonné que tous les ans, lors des Neptunalia, de l' huile fût distribuée au peuple sur le campus et dans tous les thermes et bains qui se trouvent à Côme [...] ». DEVIJVER, H. et VAN WONTERGHEM, F. : art. cit., 1981, 34 ; BOUET, A. : art. cit., « Un nouvel exemple », 1998, 111.

55. THUILLIER, J.-P. : ouv. cit., 1996, 92-93.

56. CIL I² 1529 ; CIL I, 1166; CIL X, 5807 ; ILS 5348 ; ILLRP 528 (Aletrium, Regio I) : « Lucius Betilienus Varus, fils de Lucius, s'est occupé sur décision du sénat de faire faire les installations énumérées cidessous : [...] un campus où l'on s'entraîne [...] ». DEVIJVER, H. et VAN WONTERGHEM, F. : art. cit., 1981, 38. Voir aussi Dig., XLIII, 8, 2, 9 (Ulpien), où l'on trouve l'expression in campo publico ludere.

57. BORLENGHI, A. : ouv. cit., 25-27.

58. Cic., Cael., V, 11. Voir les exercitationes campestres mentionnées plus haut.

59. MOHLER, S.L. : «The Iuvenes and Roman Education », TAPhA, 68, 1937, 442-479 (442-444); GINESTET, P. : Les organisations de la jeunesse dans l'Occident romain, Bruxelles, Latomus 213, 1991, 14 et $119-120$.

60. Hor., Carm., III, 8, 7-12. FORTUIN, R. : ouv. cit., 72-77. 
Drusus, le fils de Tibère, a fait don d' un campus à la colonie d' Issa en Dalmatie en 20 apr. J.-C. ${ }^{61}$

C'était aussi sur un campus (entre autres) que s'entraînaient les soldats ${ }^{62}$. Il s' agissait d'un espace carré ou rectangulaire ceint d' un mur, qui ne comportait comme construction qu' une tribune (tribunal en latin et $\beta \tilde{\eta} \mu \alpha$ en grec) d'où les responsables pouvaient surveiller l'exercice ou s'adresser aux troupes ${ }^{63}$. On en a retrouvé des traces archéologiques à Lambèse, à Mirebeau-sur-Bèze (Côte d'Or) et en Bretagne; ceux de Palmyre (aménagé en 183), Doura-Europos (aménagé en 208-209) et Colybrassos de Cilicie (aménagé en 288 ) sont attestés par l'épigraphie ${ }^{64}$. Il y en avait aussi un à Rome, près de la caserne des prétoriens.

Le campus, réservé aussi bien à des civils qu'à des militaires, était donc le lieu où l'on pratiquait l'exercice, autrement dit un terrain d'exercice ou de manœuvre. Des dieux présidaient-ils à l'exercice ? Un culte particulier était-il lié au campus ? Il $\mathrm{y}$ avait bien des cultes liés aux thermes ou à la palestre, lieux où étaient aussi pratiqués des jeux sportifs. Ce culte existait, pour ce qui concerne les soldats. De nombreux dieux présidaient aux exercices, dont Virtus et Disciplina, deux abstractions divinisées. Il existait également des divinités appelées campestres ${ }^{65}$. Elles étaient les

61. DEVIJVER, H. et VAN WONTERGHEM, F. : art. cit., 1985, 152 ; BOULEY, E. : « L'éducation éphébique et la formation de la "juventus" d'après quelques documents des provinces balkaniques et danubiennes », M. Garrido-Hory et A. Gonzalès (éd.), Histoire, Espaces et Marges de l'Antiquité, 1, Hommages à Monique Clavel-Lévêque, Besançon, PU Franc-Comtoises, 2003, 195-207 (203) ; BORLENGHI, A. : ouv. cit., 27-33 et 51-52.

62. Pour l'entraînement des soldats : HORSMANN, G. : Untersuchungen zur militärischen Ausbildung im republikanischen und kaiserzeitlichen Rom, Boppard a. Rh., Boldt, 1991 ; LE BOHEC, Y. : L'armée romaine sous le Haut-Empire, Paris, Picard, 2014 (3éd.), 111-126 ; LE BOHEC, Y. : art. cit., 170-187. Pour celui des cavaliers, HYLAND, A. : Training the Roman cavalry. From Arrian's Ars tactica, Douvres, A. Sutton, 1993. Pour le campus, Th. l. l., III, 1912, col. 212-221 ; DE RUGGIERO, E. : Dizionario epigrafico, II, 1, 1900, Rome, 49-52 ; DAVIES, R. : " The training Grounds of the Roman Cavalry », R. Davies, Service in the Roman Army, New York, Columbia University Press, 1989, 93-123 ; LE BOHEC, Y. : « Recherches sur les terrains d'exercice de l'armée romaine sous le Haut-Empire », Bulletin des Antiquités Luxembourgeoises, 27, 1999, 79-95 ; BORLENGHI, A. : ouv. cit., 33-39.

63. Pour les discours aux troupes, voir les discours d'Hadrien à l'armée d'Afrique : LE BOHEC, Y. (éd.) : Les discours d' Hadrien à l'armée d'Afrique. Exercitatio, Paris, De Boccard, 2003 ; SPEIDEL, M.P. : Emperor Hadrian's Speeches to the African Army. A new Text, Mayence, Verlag des Römisch-Germanischen Zentralmuseums, 2006. Ou encore Hérodien, I, 5, 2 ; II, 10, 1 ; II, 13, 35 ; VII, 8, 3 ; VIII, 7, 3, qui utilise le terme grec $\pi \varepsilon \delta i ́ o v$; Ammien Marcellin, XX, 5, 1 ; XX, 9, 6 ; XXI, 13, 9 ; HA, Max., 3, 1 ; Prob., 10,4 .

64. AE 1933, 214 ; 1931, 113 ; 1972, 636. DAVIES, R.W. : ouv. cit., 94 ; LE BOHEC, Y. : La guerre romaine, Paris, Tallandier, 2014, 167-168.

65. Pour ce passage, DE RUGGIERO, E. : Dizionario epigrafico, II, 1, 1900, Rome, 47-48 ; LE BOHEC, Y. : L'armée romaine, 2014, 125. IRBY-MASSIE, G.L. : « The Roman Army and the Cult of the Cam- 
divinités du campus et on a retrouvé un autel dédié aux Campestres et à Britannia sur le terrain d'exercice de Castlehill, en Écosse, tout comme sur celui de Newstead

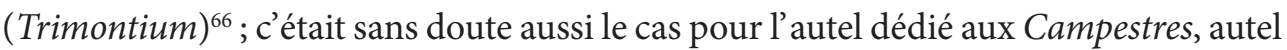
trouvé à Obernburg am Main, où se trouvait un camp militaire ${ }^{67}$. Ces divinités pouvaient être associées à d'autres dieux, souvent Epona et les Suleuiae ${ }^{68}$. Elles pouvaient aussi être désignées en Afrique par l'expression générale dii Campestres, dieux de la plaine (là où se déroulait l'exercice) ou dieux du campus ${ }^{69}$. Ce nom a sans doute été forgé sur celui de Dii Mauri, pour que ces divinités venues de l'extérieur fussent acceptées par les Romains africains ${ }^{70}$. On a retrouvé à Gemellae, en Afrique, un temple contenant deux autels dédiés aux dii Campestres. Ce temple se trouvait à l'extérieur du camp militaire et l'on peut penser qu' il était proche du terrain d'exercice, du campus, dont on n'a cependant retrouvé aucune trace ${ }^{71}$. Avec la mention d' un temple aux Matres Campestres et au Genius alae pri(mae) Hispanorum Asturum sur une inscription retrouvée à Benwell (Condercum), sur le mur d' Hadrien ${ }^{72}$, c'est le seul sanctuaire aux Campestres connu à ce jour ${ }^{73}$.

Campester peut aussi être une épithète accordée à un dieu, comme Mars, qualifié de Campester dans la péninsule Ibérique, à Tarraco ${ }^{74}$, ou encore de Némésis, qui

pestres », ZPE, 113, 1996, 293-300 (298-300) recense toutes les inscriptions mentionnant les Campestres.

66. CIL VII, 1129 ; ILS 4829 ; RIB 2135 (" Aux Campestres et à Britannia, Quintus Pisentius Iustus, préfet de la cohorte IV des Gaulois, s'est acquitté de son vœu de bon gré, avec joie et à juste titre ») et CIL VII, 1080 ; RIB I, 2121 ("Consécration aux Campestres, Aelius Marcus, décurion de l'aile Auguste des Voconces, s'est acquitté de son vœu de bon gré, avec joie et à juste titre "). DAVIES, R.W. : ouv. cit., 93 ; BIRLEY, E. : The Roman Army. Papers 1929-1986, Amsterdam, J.C. Gieben, 1998, 434 ; SPEIDEL, M.P. : "The shrine of the Dii Campestres at Gemellae », AntAfr, 27, 1991, 111-118 (107).

67. AE 2003, 1274. STEIDL, B. : " Ein Altar für die 'Campestres' aus Obernburg am Main », Bayerische Vorgeschichtsblätter, 68, 2003, 89-100.

68. CIL VII, 1114 ; RIB I, 2177 ; ILS 4831c (Bretagne, Auchendavy) : « À Mars, Minerve, aux Campestres, à Hercule, Epona, Victoire, Marcius Cocceius Firmus, centurion de la légion II Auguste ». Voir IRBY-MASSIE, G.L. : art. cit., 293.

69. HORSMANN, G. : ouv. cit., 105-107.

70. SPEIDEL, M.P. : art. cit., 1991, 118.

71. DAVIES, R.W. : ouv. cit., 118-119; SPEIDEL, M.P. : art. cit., 1991, 112 et 115.

72. CIL VII, 510 ; RIB 1334 ; ILS 4828 : « Aux Matres Campestres et au Génie de l' aile I des Astures [---] Titus [---] Agrippa, préfet, a restauré ce temple depuis le sol ». BIRLEY, E. : ouv. cit., 433-434 ; DAVIES, R.W. : ouv. cit., 93 ; SPEIDEL, M.P. : art. cit., 1991, 112.

73. SPEIDEL, M.P. : art. cit., 1991, 112.

74. CIL II, 4083 ; II/14/02, 839; ILS 2416; RIT 38 ; AE 2012, 816 : "Consécration à Mars Campester. Pour le salut de l'empereur Marc Aurèle Commode Auguste et des equites singulares, Titus Aurelius Decimus, centurion de la légion VII Gemina Heureuse, chef et en même temps maitre d'exercice (a fait ériger ce monument) et l'a dédié aux calendes de mars, sous le consulat de Mamertin et de Rufus ( $1^{\text {er }}$ mars 182)». DE RUGGIERO, E. : Dizionario epigrafico, II, 1, 1900, Rome, 48 ; SPEIDEL, M.P. : Die Equites singulares Augusti, Bonn, R. Habelt, 1965, 56. 
peut être identifiée à Fortuna ${ }^{75}$, elle aussi qualifiée de Campestris à Rome ${ }^{76}$. Ainsi que le fait remarquer E. Birley ${ }^{77}$, dans les deux cas le dédicant est un campidoctor, un maître d'exercice, ce qui souligne le lien de l'épithète portée par les deux divinités avec le campus.

Ces divinités, des déesses en réalité (on trouve aussi le nom de Matres Campestres), sont d'origine celtique et elles ne sont connues que dans la partie occidentale de l'Empire $^{78}$. C'étaient surtout les equites singulares Augusti (les gardes du corps à cheval de l'empereur) et de façon générale les cavaliers qui honoraient ces divinités, et ce sont ces gardes du corps qui ont apporté le culte des Campestres en Afrique, où elles sont donc devenues les dii Campestres $^{79}$, tout comme les cavaliers auxiliaires les ont répandues dans l' Occident romain depuis la Gaule, où elles étaient honorées à l'origine ${ }^{80}$.

À Tébessa, en Afrique, c'est une divinité proprement romaine qui est mentionnée dans une inscription d'époque flavienne : le Genius campi, le Génie du camp ${ }^{81}$.

Des cérémonies religieuses se déroulaient sur le campus. C'est ainsi que tous les ans, le 3 janvier, le commandant dédiait un nouvel autel à Jupiter Optimus Maximus, l'ancien ayant été enterré ${ }^{82}$. Il y avait un temple sur le terrain d'exercice de Doura Europos $^{83}$, tout comme semble-t-il sur le terrain d'exercice des cohortes prétoriennes et urbaines à Rome. Les equites singulares, souvent pour commémorer leur démobilisation, dressaient un autel en marbre, autel dédié aux Campestres, à Jupiter Optimus Maximus, à Junon, Minerve, Mars, Victoire, Hercule, Fortune et au genius singularium Augusti entre autres ${ }^{84}$.

Qu'en était-il chez les civils ? Si des cultes étaient célébrés sur le campus, cultes qui n'étaient pas en lien étroit avec lui, il n'y avait pas de culte particulier lié aux activités sportives qui y étaient pratiquées. Il y avait sur le Champ de Mars (c'était

75. DE RUGGIERO, E. : Dizionario epigrafico, II, 1, 1900, Rome, 48.

76. CIL VI, 533 ; ILS 2088 : «À la sainte Némésis Campestris, pour le salut de nos deux maîtres les empereurs. Publius Aelius Pacatius, fils de Publius, inscrit dans la tribu Aelia et originaire de Scupi. Il avait promis de le faire quand il était docteur de cohorte, et maintenant qu' il est maitre d'exercice de la cohorte I prétorienne pieuse et vengeresse, prévenu en songe, il a de bon cœur et joyeusement fait placer (cette dédicace) ». SPEIDEL, M.P. : ouv. cit., 56.

77. BIRLEY, E. : ouv. cit., 404.

78. BIRLEY, E. : ouv. cit., 419-421 ; SPEIDEL, M.P. : art. cit., 1991, 107.

79. SPEIDEL, M.P. : ouv. cit., 56-57 ; SPEIDEL, M.P. : art. cit., 1991, 117-118 ; IRBY-MASSIE, G.L. : art. cit., 294-296.

80. IRBY-MASSIE, G.L. : art. cit., 294-296.

81. ILAlg. I, 3596 ; LE BOHEC, Y. : La troisième légion Auguste, Paris, CNRS, 1989, 362.

82. RICHMOND, I.A. : « The Roman army and Roman religion », Bulletin of the John Rylands Library, 45, 1962, 185-197 (187-189) ; DAVIES, R.W. : ouv. cit., 98.

83. AE 1931, 113 : la cohors II Vlpia equitata ciuium Romanorum sagittariorum a construit un temple et érigé une statue lors de l'agrandissement du campus. DAVIES, R.W. : ouv. cit., 94.

84. IRBY-MASSIE, G.L. : art. cit., 295. 
du reste la raison de son nom) un autel de Mars, un sanctuaire très ancien, lié à la fonction militaire du campus ${ }^{85}$. Des cultes étaient liés à la présence de cet autel, mais ils étaient tous en relation avec la guerre et pas avec la pratique des exercices, qu' ils fussent une préparation à la guerre ou pas. Il en allait de même pour les innombrables sanctuaires, temples, autels qui s'y trouvaient également et qui n'étaient pas sur le terrain d'exercices proprement dit. À l'extérieur de Rome, il pouvait y avoir consécration d' un campus pour le salut d' un empereur ${ }^{86}$ ou consécration d' un campus et de son portique au numen de l'Auguste et à Vulcain ${ }^{87}$. Un campus pouvait être dédié au Génie de la colonie et des colons ${ }^{88}$. De façon générale, on trouvait sur le campus des tombeaux, des statues, des chapelles et des autels (sacella, arae) ${ }^{89}$. Mais il n'y avait pas de dédicace au Génie du campus, comme il y en avait pour le campus à usage militaire, et aucune dédicace n'a été faite aux divinités Campestres.

Les associations de la Jeunesse célébraient des cultes, cette célébration était même une de leurs occupations principales ${ }^{90}$. Ainsi que le souligne P. Ginestet, ils se disaient souvent les cultores d' un dieu ou encore les iuuenes d' un dieu. Parmi ces dieux, le plus fréquemment honoré en Italie était Hercule ${ }^{91}$. Venaient ensuite le culte de l'empereur divinisé, celui de Jupiter, Diane, Iuuentus, Junon, Apollon, Vénus, Minerve, Éros et de leur Génie ${ }^{92}$. Dans les provinces, le culte le plus répandu était le culte impérial, puis celui de Jupiter, Hercule, Diane, Mars, Iuuentus et de divinités mineures et " locales " ainsi que celui du Génie du collège ${ }^{93}$. Ces cultes, y compris

85. COARELLI, F. : ouv. cit., 188.

86. DEVIJVER, H. et VAN WONTERGHEM, F. : art. cit., 1992, 1048. Sur le territoire de Clunia, uicus de Venia, en Tarraconaise. Il s'agit de Trajan. BOUET, A. : art. cit., 1999, 464. Pour les cultes de façon générale, BORLENGHI, A. : ouv. cit., 170-173.

87. CIL XIII, 3107 (Nantes). BOUET, A. : art. cit., 1999, 464. Voir aussi CIL XII, 4338 et BOUET, A. : ouv. cit., 2003, t. II, 178.

88. CIL X, 1236 ; ILS 5392 ; ILLRP 116 (Nola, Regio I). DEVIJVER, H. et VAN WONTERGHEM, F. : art. cit., 1981, 37.

89. CIL X, 1781 ; CIL X, 1793 ; CIL I, 698 ; ILLRP 518 ; ILS 5317 ; ILS 5389, Puteoli, Regio I. DEVIJVER, H. et VAN WONTERGHEM, F. : art. cit., 1981, 44-45 ; DEVIJVER, H. et VAN WONTERGHEM, F. : art. cit., 1985, 150 ; BORLENGHI, A. : ouv. cit., 51. Pour les cultes pratiqués sur le campus, voir la communication de Beatriz Pañeda dans ce même volume.

90. MOHLER, S.L. : art. cit., 472-473 ; JACZYNOWSKA, M. : Les associations de la jeunesse romaine sous le Haut-Empire, Wroclaw, 1978, 55 et 62 ; LADAGE, D. : « Collegia iuvenum. Ausbildung einer municipalen Elite? », Chiron, 9, 1979, 319-346 (339-341 et 345-346) ; GINESTET, P. : ouv. cit., 1991, 168.

91. GINESTET, P. : ouv. cit., 1991, 172 ; BOUET, A. : art. cit., 1999, 479. Pour le culte d'Hercule, JACZYNOWSKA, M. : " Le culte de l' Hercule romain au temps du Haut-Empire », ANRW II, 17, 2, 631-661.

92. GINESTET, P. : ouv. cit., 1991, 172 ; BOUET, A. : art. cit., 1999, 478-479.

93. GINESTET, P. : ouv. cit., 1991, 173-175. 
celui d'Hercule, n'étaient pas accomplis dans le cadre du campus, mais dans le cadre du collège, et n'étaient pas liés à la pratique sportive.

La pratique sportive constituait un élément important de l'éducation des jeunes gens, aussi bien à Rome que dans le reste de l'Empire, à tel point du reste que sans elle, l'éducation était incomplète. Le lieu par excellence de la pratique sportive et de l'exercice, à Rome comme dans les provinces occidentales, était le campus. Des cultes y étaient pratiqués. Chez les militaires, ces cultes étaient en lien direct avec le lieu et l'activité qui s'y déroulait, puisqu' ils s'adressaient à des divinités qui présidaient aux exercices. Chez les civils, il n'en était rien. Il n'y avait pas de divinités campestres, aucune divinité particulière ne présidait à ces exercices et aucun culte, par conséquent, ne lui était associé. 